\title{
Integrating Ema, Clinical Assessment and Wearable Sensors to Examine the Association between Major Depressive Disorder (MDD) and Alcohol Use
}

Paola Pedrelli ${ }^{1,2}, \mathrm{PhD}$; Esther Howe ${ }^{1}$, BA; David Mischoulon ${ }^{1,2}, \mathrm{MD}, \mathrm{PhD}$; Rosalind Picard ${ }^{3,4}$, ScD, FIEE; Asma Ghandeharioun $^{3}$, MA; Szymon Fedor ${ }^{3,5}$, PhD

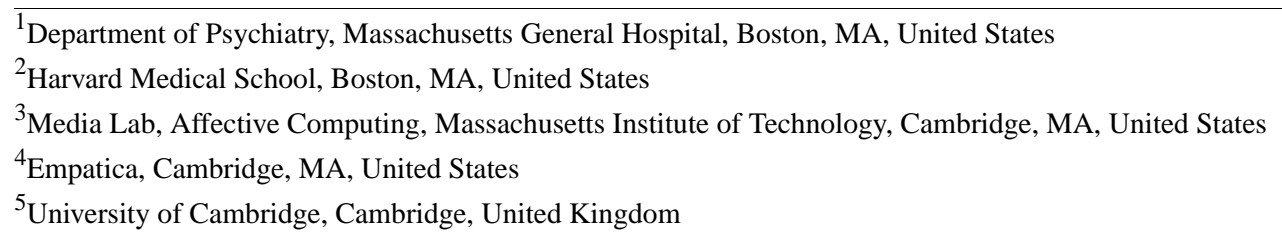

Corresponding Author:

Paola Pedrelli, PhD

Department of Psychiatry

Massachusetts General Hospital

6th Floor

One Bowdoin Square

Boston, MA,

United States

Phone: 16177243678

Email: ppedrelli@mgh.harvard.edu

\section{Abstract}

Background: Depression is the leading cause of disability worldwide. Heavy drinking often co-occurs with Major Depressive Disorder (MDD), preventing the amelioration of symptoms and increasing disability. New technology-based assessment tools such as ecological momentary assessment (EMA) and wearable sensors provide the opportunity for a more detailed examination of the interplay between these two conditions. While the association between low mood and heavy drinking has been extensively examined, multi-method assessments including EMA, sensors and clinician-rated measures have not been utilized to study the association between depression and heavy alcohol use.

Objective: To examine the association between depressive symptoms and alcohol consumption by integrating multiple sources of data including EMA, clinical assessment, and wearable sensors.

Methods: Individuals with MDD complete an 8-week protocol that involves tracking depressive symptoms and alcohol consumption daily through an EMA. Mood is captured via surveys delivered twice daily that include 10 items of the Positive and Negative Affect Scale (PANAS) assessing negative affect (NA) and positive affect (PA). Participants wear Empatica E4 wristband sensors that track electrodermal activity (EDA) and accelerometer data 23 hours/day. The clinician-rated Hamilton Depression Rating Scale (HDRS) is administered biweekly to assess depressive symptoms. MovisensXS, the app delivering the EMA, tracks text messages, phone calls, location, app usage, and screen on/off behavior.

Results: To date, 16 of 30 projected participants have completed the study. All participants are expected to complete the study by 10/2017. Preliminary analyses confirmed the accuracy of the daily mood ratings. There was a significant linear relationship between NA/PA ratio from EMA ratings and the clinician-based ratings $(P=1.3 \mathrm{e}-6)$. To focus on the association of low mood and drinking behavior, we solely included instances where NA $>=P A$ and observed a significant association $(P=0.001)$ between low mood (as a ratio of total NA divided by PA) and higher alcohol use. Analyses will be repeated for all 30 participants. E4 accelerometer data and location data will help elucidate whether mobility moderates the association between mood and depression, such that individuals who drink at home may exhibit greater depressive symptomatology. Finally, the association between EDA and alcohol use will be examined. Final results will be presented at the Connected Health Conference.

Conclusions: To date, results show a significant association between low mood and alcohol consumption. Results of planned analyses will further clarify the temporal association between mood and alcohol use among depressed patients, and possible moderators and mediators of this relationship. A precise understanding of the association between low mood, physiological states 
and heavy drinking will facilitate the development of "just-in-time" ecological momentary interventions for both reduction of depressed mood and heavy drinking.

(iproc 2017;3(1):e51) doi: 10.2196/iproc.8584

\section{KEYWORDS}

alcohol; Depression; ecological momentary assessment; sensors

\section{Multimedia Appendix 1}

Full poster.

\section{[PDF File (Adobe PDF File), 430KB-Multimedia Appendix 1]}

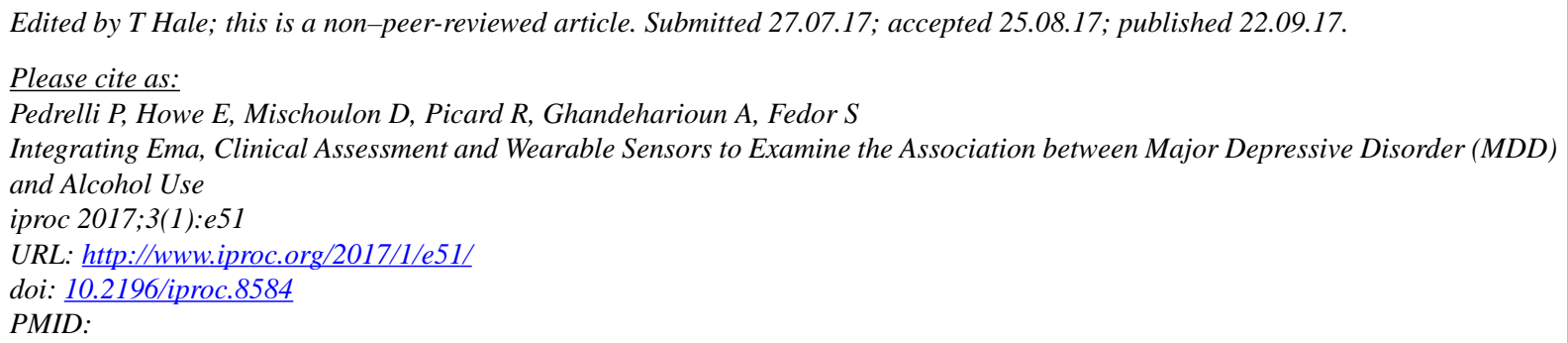

(CPaola Pedrelli, Esther Howe, David Mischoulon, Rosalind Picard, Asma Ghandeharioun, Szymon Fedor. Originally published in Iproceedings (http://www.iproc.org), 22.09.2017. This is an open-access article distributed under the terms of the Creative Commons Attribution License (https://creativecommons.org/licenses/by/4.0/), which permits unrestricted use, distribution, and reproduction in any medium, provided the original work, first published in Iproceedings, is properly cited. The complete bibliographic information, a link to the original publication on http://www.iproc.org/, as well as this copyright and license information must be included. 